\title{
High-resolution view of compound promiscuity [version 1;
}

\section{peer review: 3 approved]}

\section{Ye Hu, Jürgen Bajorath (iD}

Department of Life Science Informatics, B-IT, LIMES Program Unit Chemical Biology and Medicinal Chemistry, Rheinische FriedrichWilhelms-Universität, Bonn, Germany

V1 First published: 27 Jun 2013, 2:144

https://doi.org/10.12688/f1000research.2-144.v1

Latest published: 26 Jul 2013, 2:144

https://doi.org/10.12688/f1000research.2-144.v2

\section{Abstract}

Compound promiscuity is defined as the ability of a small molecule to specifically interact with multiple biological targets. So-defined promiscuity is relevant for drug discovery because it provides the molecular basis of polypharmacology, which is increasingly implicated in the therapeutic efficacy of drugs. Recent studies have analyzed different aspects of compound promiscuity on the basis of currently available activity data. In this commentary, we present take-home messages from these studies augmented with new results to generate a detailed picture of compound promiscuity that might serve as a reference for further discussions and research activities.

Keywords

polypharmacology, compound promiscuity, drug efficiacy
Open Peer Review

Approval Status

1

2

3

version 2

(update)

26 Jul 2013

version 1

27 Jun 2013
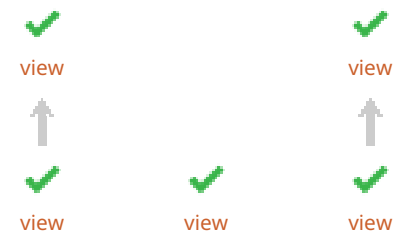

1. Stefan Laufer, University of Tübingen,

Tübingen, Germany

2. Hans Matter, Sanofi-Aventis, Paris, France

3. Jeremy L. Jenkins, Novartis Institutes for

BioMedical Research, MA, USA

Any reports and responses or comments on the article can be found at the end of the article.

Corresponding author: Jürgen Bajorath (bajorath@bit.uni-bonn.de)

Competing interests: No competing interests were disclosed.

Grant information: The author(s) declared that no grants were involved in supporting this work.

Copyright: ( $) 2013 \mathrm{Hu} \mathrm{Y}$ and Bajorath J. This is an open access article distributed under the terms of the Creative Commons Attribution License, which permits unrestricted use, distribution, and reproduction in any medium, provided the original work is properly cited.

How to cite this article: $\mathrm{Hu} \mathrm{Y}$ and Bajorath J. High-resolution view of compound promiscuity [version 1; peer review: 3 approved] F1000Research 2013, 2:144 https://doi.org/10.12688/f1000research.2-144.v1

First published: 27 Jun 2013, 2:144 https://doi.org/10.12688/f1000research.2-144.v1 


\section{Introduction}

Polypharmacology is an emerging theme in drug discovery ${ }^{1,2}$. It is generally accepted that drugs often elicit their therapeutic effects through interactions with different targets and the ensuing modulation of multiple signaling pathways. In some therapeutic areas such as oncology, polypharmacology is heavily exploited, for example, through the use of promiscuous ATP site-directed protein kinase inhibitors ${ }^{3}$. In other areas, such as the treatment of infectious or chronic inflammatory diseases, achieving a high degree of target selectivity of drug candidates plays a major role.

The study of drug polypharmacology has become an important topic in pharmaceutical research ${ }^{4,5}$, especially focusing on combined computational and experimental analysis ${ }^{5}$. On the basis of drug-target networks, it was estimated early on that a drug interacts on average with approximately two targets ${ }^{4}$. More recent estimates from computational data analysis suggest that drugs might bind on average to two to seven targets, depending on the primary target families, and that more than $50 \%$ of current drugs might interact with more than five targets ${ }^{6}$.

Compound promiscuity as defined herein is the origin of polypharmacology. Promiscuity analysis can be extended from drugs to bioactive compounds through computational mining of currently available activity data. The results of activity data analysis are generally affected by data incompleteness ${ }^{7}$. This potential influence can only be eliminated by reaching the ultimate (and probably elusive) goal of chemogenomics ${ }^{8}$, i.e., testing all compounds against all targets. In the presence of data incompleteness, compound promiscuity rates are likely underestimated. However, it is not certain that further increasing amounts of assay data will indeed significantly alter the currently emerging view of compound promiscuity (vide infra).

Recent studies have generated a differentiated picture of compound promiscuity. The interested reader is also referred to comprehensive reviews of compound promiscuity analysis ${ }^{9}$ and polypharmacology ${ }^{6}$. In this commentary, we summarize key messages from recent promiscuity analysis in a compact format. It is hoped that this summary might be helpful as a reference for further studies.

\section{Key results of compound promiscuity analysis}

Public data sources for compound promiscuity analysis discussed herein have been $\mathrm{ChEMBL}^{10}$, the major repository of compound activity data from medicinal chemistry (currently in May 2013 containing $1,295,510$ compounds with a total of $11,420,351$ activity annotations), the PubChem BioAssay database ${ }^{11}$, the major repository of screening data (with more than 3300 confirmatory assays), and DrugBank ${ }^{12}$, which currently contains 1518 approved and 5080 experimental drugs.

It is important to note that collecting all activity annotations for a compound reported in the literature including, for example, reporter gene or other cell-based assays is at best providing a measure of assay promiscuity, but not of specific interactions with different targets ${ }^{9}$. Therefore, it is generally required to apply data confidence criteria such as the presence of well-defined activity measurements or evidence for direct ligand-target interactions ${ }^{9}$ (as provided in ChEMBL as activity data filters).

\section{Activity measurement dependence}

When monitoring the growth of compound activity data in ChEMBL over a period of more than two years from its original release (January 2010) to release 13 (May 2012), a significant increase in the number of promiscuous compounds was detected ${ }^{13}$. However, by quantifying compound-based target relationships, it was determined that the increase in compounds with activity against targets from different families was largely due to (assay-dependent) $I C_{50}$ measurements, rather than (assay-independent) equilibrium constants $\left(K_{i} \text { values }\right)^{13} . \mathrm{IC}_{50}$ values are easier to determine than $\mathrm{K}_{\mathrm{i}}$ values and provide the readout of most primary biochemical assays (except single-point screening assays), which might at least in part rationalize greater target coverage and the $\mathrm{IC}_{50}$-dependent increase in compound promiscuity across different families. However, it can also not be excluded that apparent promiscuity in different assays is higher on the basis of $\mathrm{IC}_{50}$ measurements, given their assay dependence (and often limited accuracy). Regardless, the type of activity measurements that are taken into account influences the outcome of promiscuity analysis. Thus, clear specification of activity measurements and data selection criteria are required.

The subset of compounds with available $\mathrm{K}_{\mathrm{i}}$ measurements from ChEMBL release 13 was further investigated. On the basis of $K_{i}$ measurements, approximately $62 \%$ of all compounds were only annotated with a single target, $\sim 36 \%$ with two or more targets from the same family, and only $2 \%$ of all active compounds with multiple targets from different families ${ }^{14}$. A promiscuous bioactive compound was found to interact on average with two to three targets.

\section{Activity data from different sources}

One might anticipate that the degree of compound promiscuity would be particularly high in screening assays (even if frequent hitters and other non-specific compounds are excluded). Therefore, 1085 confirmatory bioassays from PubChem were systematically analyzed. It was found that $\sim 77 \%$ of all confirmed active compounds were tested in more than 50 different assays ${ }^{15}$. Thus, these active PubChem compounds provided a sound basis for promiscuity assessment. These results were in part surprising. An active PubChem compound displayed a $\sim 50 \%$ probability to interact with two or more targets. The probability to interact with more than five targets was only $\sim 8 \%$. On average, a PubChem screening hit was active against 2.5 targets. For comparison, compounds from the $I_{50}-$ and $K_{-}^{-}$-based subsets of ChEMBL release 14 (August 2012) interacted on average with 1.4 and 1.7 targets, respectively ${ }^{15}$. The comparably low ratios observed for both compound subsets indicated that $\mathrm{IC}_{50}$ measurements did not systematically increase promiscuity rates (vide supra). The analysis of active compounds from PubChem confirmatory assays provided an upper level estimate of promiscuity, which was not significantly higher than that for ChEMBL compounds.

\section{Prevalent promiscuity profile}

Detailed analysis of compound activity data from ChEMBL release 14 (August 2012) has made it possible to derive a promiscuity profile that is most characteristic of bioactive compounds from medicinal 
chemistry sources. The majority of currently available promiscuous compounds is active in the sub- $\mu M$ range against two to five targets from the same family and displays potency differences against these targets within one or two orders of magnitude ${ }^{16}$. An important aspect of this representative profile is that promiscuity does not imply low potency. Furthermore, compounds that are highly potent against a (primary) target and weakly potent against others are not frequently found ${ }^{16}$.

\section{Up-to-date promiscuity rates}

In Table 1, current average promiscuity rates are summarized for compounds from ChEMBL, PubChem, and DrugBank. For promiscuity assessment of drugs, all targets reported in DrugBank were considered.

If all compounds with single or multiple target annotations are analyzed, ChEMBL compounds interact on average with one to two targets and PubChem compounds with two to three. However, approved drugs have on average close to six targets. In contrast, the degree of promiscuity of experimental drugs is considerably lower, with less than two targets per drug candidate. If only promiscuous compounds or drugs are taken into account (i.e., if compounds with single target annotations are excluded), promiscuity rates only slightly increase by about one target per compound, the exception being experimental drugs whose average number of targets increases from 1.8 to 4.7 .

In Table 2, the probability of promiscuity is reported for compounds from different sources (calculated from target distributions of compounds). For a ChEMBL compound with available $\mathrm{IC}_{50}$ and $\mathrm{K}_{\mathrm{i}}$ measurements, the current probability of activity against two or more targets is $\sim 25 \%$ and $\sim 38 \%$, respectively (if both $\mathrm{IC}_{50}$ and $\mathrm{K}_{\mathrm{i}}$ measurements were available for a compound, they were separately considered). However, for activity against more than five targets, the probabilities are reduced to only $\sim 1 \%$. Similar observations are made for confirmed PubChem screening hits (providing an upper-limit promiscuity assessment for bioactive compounds, vide supra). In this case, the probability of activity against two or more, or against more than five targets is $\sim 51 \%$ and $\sim 8 \%$, respectively. Furthermore, the probability of promiscuity of approved drugs from DrugBank is $\sim 84 \%$ and the probability to interact with more than five targets still $\sim 37 \%$. For experimental drugs, the corresponding probabilities are much lower, with only $\sim 24 \%$ and $\sim 3 \%$, respectively.

\section{Conclusions}

Herein, we have provided a detailed and up-to-date view of compound promiscuity, the molecular basis of polypharmacology. For active compounds from medicinal chemistry and biological screening sources, the degree of promiscuity is lower than for drugs. There is a notable increase in promiscuity from bioactive compounds over drug candidates to approved drugs. The exploration of possible reasons for this apparent "promiscuity enrichment" along the drug discovery pathway should provide interesting opportunities for future research. On the basis of currently available high-confidence activity data, promiscuity of bioactive compounds is limited (and very low across different target families). However, if compounds are promiscuous, they typically bind to their targets with relatively high potency. Given the overall low degree of promiscuity of bioactive compounds including screening hits in the presence of nearly exponential data growth in recent years, it remains an open question if future chemogenomics efforts might substantially change the current picture of compound promiscuity (vide supra). The majority of available bioactive compounds have single target annotations and we believe it is unlikely that most of them will display a high degree of currently undiscovered promiscuity. Hence, we would also conclude that the target specificity paradigm that has long dominated small molecule discovery efforts should continue to play a major

Table 1. Average promiscuity of different compound categories.

\begin{tabular}{lll} 
Compound categories & & Avg. \# targets/compound \\
ChEMBL 14/all bioactive compounds & $\mathbf{K}_{\mathbf{i}}$ & 1.7 \\
& IC $_{50}$ & 1.4 \\
DrugBank/drugs & Approved & 5.9 \\
PubChem/active compounds & Experimental & 1.8 \\
\hline ChEMBL 14/promiscuous compounds & & 2.5 \\
\hline DrugBank/promiscuous drugs & $\boldsymbol{K}_{\boldsymbol{i}}$ & 2.9 \\
\hline PubChem/promiscuous active compounds & IC $_{50}$ & 2.7 \\
\hline
\end{tabular}

The average number of targets is reported for compounds from ChEMBL release 14 (divided into $\mathrm{K}_{\mathrm{i}}$ and $\mathrm{IC}_{50}$ valuebased subsets), approved or experimental drugs from DrugBank 3.0, and active compounds from PubChem confirmatory bioassays. Corresponding statistics are provided in italics for promiscuous compounds (having two or more target annotations). For compounds from ChEMBL, only high-confidence activity annotations were taken into account (i.e., explicit activity measurements with the highest confidence level of direct ligand-target interactions). For calculations on drugs, all DrugBank target categories were taken into account. 
Table 2. Probability of promiscuity.

\begin{tabular}{llll} 
Compound categories & & \# Targets & Probability (\%) \\
ChEMBL 14/all bioactive compounds & $\mathbf{K}_{\mathbf{i}}$ & $\mathbf{2}$ & 37.9 \\
& & $\mathbf{5}$ & 1.2 \\
& $\mathbf{I C}_{\mathbf{5 0}}$ & $\mathbf{2}$ & 24.7 \\
& & $\mathbf{2}$ & 0.8 \\
\hline DrugBank/drugs & Approved & $\mathbf{2}$ & 84.1 \\
& & $\mathbf{2 5}$ & 37.4 \\
\hline PubChem/active compounds & Experimental & $\mathbf{2}$ & 23.6 \\
\hline & & $\mathbf{5}$ & 3.4 \\
\hline
\end{tabular}

For different compound categories and activity measurements, the probability of a compound to be active against two or more targets or more than five targets is reported.

role, despite emerging "anti-reductionism" and the increasing focus on phenotypic readouts.

Author contributions

JB conceived the study, YH collected and organized the data and information, YH and JB wrote the manuscript.

\section{Competing interests}

No competing interests were disclosed.

\section{Grant information}

The author(s) declared that no grants were involved in supporting this work.
1. Paolini GV, Shapland RH, van Hoorn WP, et al:: Global mapping of pharmacological space. Nat Biotechnol. 2006; 24(7): 805-815. PubMed Abstract | Publisher Full Text

2. Boran $A D$, lyengar $R$ : Systems approaches to polypharmacology and drug discovery. Curr Opin Drug Discov Devel. 2010; 13(3): 297-309. PubMed Abstract | Free Full Text

3. Knight ZA, Lin H, Shokat KM: Targeting the cancer kinome through polypharmacology. Nat Rev Cancer. 2010; 10(2): 130-137. PubMed Abstract | Publisher Full Text | Free Full Text

4. Yildirim MA, Goh KI, Cusick ME, et al.: Drug-target network. Nat Biotechnol. 2007; 25(10): 1119-1126

PubMed Abstract | Publisher Full Text

5. Lounkine E, Keiser MJ, Whitebread S, et al.: Large-scale prediction and testing of drug activity on side-effect targets. Nature. 2012; 486(7403): 361-367. PubMed Abstract | Publisher Full Text | Free Full Text

6. Jalencas $\mathrm{X}$, Mestres J: On the origins of drug polypharmacology. Med Chem Comm. 2013; 4(1): 80-87. Publisher Full Text

7. Mestres J, Gregori-Puigjané E, Valverde S, et al: Data completeness--the Achilles heel of drug-target networks. Nat Biotechnol. 2008; 26(9): 983-984. PubMed Abstract | Publisher Full Text

8. Rognan D: Chemogenomic approaches to rational drug design. $\mathrm{Br} J$ Pharmacol. 2007; 152(1): 38-52.

PubMed Abstract | Publisher Full Text | Free Full Text

9. Hu Y, Bajorath J: Compound promiscuity: what can we learn from current data? Drug Discov Today. 2013; 18(13-14): 644-650.

PubMed Abstract | Publisher Full Text
10. Gaulton A, Bellis LJ, Bento AP, et al:: ChEMBL: a large-scale bioactivity database for drug discovery. Nucleic Acids Res. 2012; 40(Database issue): D1100-D1107.

PubMed Abstract | Publisher Full Text | Free Full Text

11. Wang Y, Xiao J, Suzek TO, et al.: PubChem's bioassay database. Nucleic Acids Res. 2012; 40(Database issue): D400-D412.

PubMed Abstract | Publisher Full Text | Free Full Text

12. Knox C, Law V, Jewison $\mathrm{T}$, et al.: DrugBank 3.0: a comprehensive resource for 'omics' research on drugs. Nucleic Acids Res. 2011; 39(Database issue): D1035-41.

PubMed Abstract | Publisher Full Text | Free Full Text

13. Hu Y, Bajorath $\mathrm{J}$ : Growth of ligand-target interaction data in ChEMBL is associated with increasing and activity measurement-dependent compound promiscuity. J Chem Inf Model. 2012; 52(10): 2550-2558. PubMed Abstract | Publisher Full Text

14. Hu Y, Bajorath J: How promiscuous are pharmaceutically relevant compounds? A data-driven assessment. AAPS J. 2013; 15(1): 104-111. PubMed Abstract | Publisher Full Text | Free Full Text

15. Hu Y, Bajorath $\mathrm{J}$ : What is the likelihood of an active compound to be promiscuous? Systematic assessment of compound promiscuity on the basis of PubChem confirmatory bioassay data. AAPS J. 2013; 15(3): 808-15. PubMed Abstract | Publisher Full Text | Free Full Text

16. Hu Y, Bajorath J: Promiscuity profiles of bioactive compounds: potency range and difference distributions and the relation to target numbers and families, in revision (the reference status will be updated as soon as possible). Med Chem Commun. 2013; 4: 1196-1201.

Publisher Full Text 


\section{Open Peer Review}

\section{Current Peer Review Status:}

\section{Version 1}

Reviewer Report 16 July 2013

https://doi.org/10.5256/f1000research.1780.r1067

(C) 2013 Jenkins J. This is an open access peer review report distributed under the terms of the Creative Commons Attribution License, which permits unrestricted use, distribution, and reproduction in any medium, provided the original work is properly cited.

\section{Jeremy L. Jenkins}

Centre for Protein Chemistry, Novartis Institutes for BioMedical Research, MA, USA

$\mathrm{Hu}$ and Bajorath's update on compound promiscuity in public compound bioactivity databases is timely with the increasing cognizance of polypharmacology and its role in the efficacy and safety of drugs. The article aims to raise reader awareness and present new questions to be answered, and so the title, abstract, and content are appropriate. All data are freely available for download for primary sources mentioned.

The conclusions are fair and unbiased.

Additional questions do arise from this survey. First, does the average number of targets per compound differ from the median (or do highly promiscuous compounds skew the average?). Second, is it reasonable to begin distinguishing promiscuous from privileged compounds? For example, by incorporating target class information, staurosporine might be viewed differently from quercetin, where the former represents a highly privileged scaffold among kinases and the latter displays IC50 values against an abundance of target types.

Third, the drug discovery field needs to understand if the "promiscuity enrichment" that occurs between the screening hits phase to the marketed drug phase largely reflects the depth of bioactivity data coverage for drugs, as drugs are highly profiled globally. The hit rates of drugs and medchem compounds across the same set of assays and targets would be needed to definitively conclude that drugs are more promiscuous.

However, the apparent increased promiscuity of drugs supports the growing resurgence of phenotypic screening, the impetus for exploring compound combinations in the context of multiple genotypes, and begs the question of how medchem optimization of multiple targets should be attacked.

Competing Interests: No competing interests were disclosed.

I confirm that I have read this submission and believe that I have an appropriate level of expertise to confirm that it is of an acceptable scientific standard. 
Reviewer Report 09 July 2013

https://doi.org/10.5256/f1000research.1780.r1052

(c) 2013 Matter H. This is an open access peer review report distributed under the terms of the Creative Commons Attribution License, which permits unrestricted use, distribution, and reproduction in any medium, provided the original work is properly cited.

\section{Hans Matter}

Sanofi-Aventis, Paris, France

This interesting manuscript presents a view on compound promiscuity based on in-vitro data and the number of potential targets per compound in public databases such as ChEMBL, PubChem and DrugBank. In particular the authors investigate and challenge the notion that most compounds today in lead findings are active on a large multitude of biological data. The title is appropriate for this contribution and the abstract sufficiently summarizes this study. The conclusions are balanced and justified on the basis of the data analysis; this is therefore an essential view on the number of targets.

It is an interesting observation from this study that DrugBank annotated drugs appear to interact with a higher number of molecular targets compared to early phase compounds in ChEMBL or PubChem. Any interpretation of this finding should be treated with caution, but it is tempting to discuss from a partially historical view as DrugBank may be enriched with older drugs that would have been subjected to less strict requirements for in-vitro selectivity than in today's drug discovery. In addition during and after approval, drugs may have been tested in more profiling assays as is the case with earlier screening-type substances.

Following the authors, this interesting argument also supports target-specific drug discovery paradigms used in past years. However, working with public databases leads to many caveats, all of which have been pointed out earlier, e.g. incompleteness of the data matrix and differences of data from different sources. It might be interesting for future investigations to cross-check this conclusion for compounds targeting families like kinases or GPCRs. Due to the challenges of inherent selectivity in those families one could expect a larger percentage of promiscuous compounds. The same discrimination might possibly be true for smaller versus larger compounds.

Competing Interests: No competing interests were disclosed.

\section{I confirm that I have read this submission and believe that I have an appropriate level of expertise to confirm that it is of an acceptable scientific standard.}

Reviewer Report 01 July 2013

https://doi.org/10.5256/f1000research.1780.r1036 
(c) 2013 Laufer S. This is an open access peer review report distributed under the terms of the Creative Commons Attribution License, which permits unrestricted use, distribution, and reproduction in any medium, provided the original work is properly cited.

\section{Stefan Laufer}

University of Tübingen, Tübingen, Germany

Excellent work. My field ("the kinase community") will benefit a lot form this commentary as compound promiscuity is an issue.

Competing Interests: No competing interests were disclosed.

I confirm that I have read this submission and believe that I have an appropriate level of expertise to confirm that it is of an acceptable scientific standard.

\section{Comments on this article}

\section{Version 1}

Author Response ( F1000Research Advisory Board Member ) 08 Jul 2013

Jürgen Bajorath, Rheinische Friedrich-Wilhelms-Universität, Bonn, Germany

Updates are available for the following references:

9. Drug Discov Today (2013); 18 (13-14): 644-650.

15. AAPS J. (2013); 15 (3): 808-815

16. Med. Chem. Commun. (2013), in press.

Competing Interests: No competing interests were disclosed. 
The benefits of publishing with F1000Research:

- Your article is published within days, with no editorial bias

- You can publish traditional articles, null/negative results, case reports, data notes and more

- The peer review process is transparent and collaborative

- Your article is indexed in PubMed after passing peer review

- Dedicated customer support at every stage

For pre-submission enquiries, contact research@f1000.com 